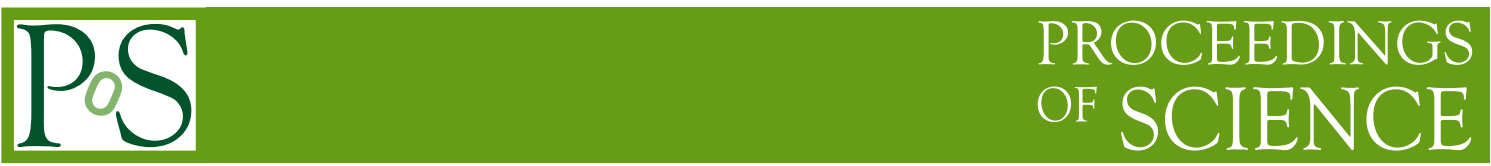

\title{
Moments of Generalized Tensor Parton Distributions
}

\section{Meinulf Göckeler ${ }^{a}$, Philipp Hägler $^{b}$, Roger Horsley ${ }^{c}$, Dirk Pleiter ${ }^{d}$, Paul E. L. Rakow ${ }^{e}$,} Andreas Schäfer ${ }^{* a}$, Gerrit Schierholz ${ }^{d f}$, and James M. Zanotti ${ }^{d}$

a Institut für Theoretische Physik, Universität Regensburg, 93040 Regensburg, Germany

${ }^{b}$ Department of Physics and Astronomy, Vrije Universiteit, 1081 HV Amsterdam, The Netherlands

${ }^{c}$ School of Physics, University of Edinburgh, Edinburgh EH9 3JZ, UK

d John von Neumann-Institut für Computing NIC, Deutsches Elektronen-Synchrotron DESY, 15738 Zeuthen, Germany

${ }^{e}$ Theoretical Physics Division, Department of Mathematical Sciences, University of Liverpool, Liverpool L69 3BX, UK

${ }^{f}$ Deutsches Elektronen-Synchrotron DESY, 22603 Hamburg, Germany

E-mail: andreas.schaefer@physik.uni-r.de

\section{QCDSF-UKQCD Collaboration}

First lattice results on moments of the tensor GPDs are presented. These quantities are highly relevant for the nucleon spin structure as they describe the correlation between quark spin, nucleon spin and quark orbital angular momentum. The Soffer bound is shown to be nearly saturated for the up and down quarks in a proton.

XXIIIrd International Symposium on Lattice Field Theory

25-30 July 2005

Trinity College, Dublin, Ireland

\footnotetext{
* Speaker.
} 


\section{Introduction: What are Tensor GPDs?}

Generalized Parton Distributions (GPDs) have in recent years evolved into the standard comprehensive formalism to analyse inclusive and exclusive processes on the same theoretical footing and to combine the information content of different measurements in the most efficient manner. (For a very nice review see [1].) Because they contain such a wealth of information GPDs have a potentially highly non-trivial functional form and there exist many different ones, e.g. eight for a spin $\frac{1}{2}$ hadron like the nucleon. Therefore, although there are many different experiments which provide information on GPDs additional input from lattice calculations is indispensable to pin down their shape. This is especially true for tensor GPDs for which direct experimental information is very hard to obtain. Our calculations for moments of tensor GPDs [2] should be seen as part of the comprehensive QCDSF programme to study the structure of hadrons. The results should be trusted to the extent to which the results agree with experimental results for those GPDs for which the latter are available.

GPDs are response functions of hadrons: some quark or gluon with longitudinal momentum fraction $x+\xi$ is replaced by one with longitudinal momentum $x-\xi$ plus possibly transverse momentum. The precise definition of the tensor GPDs of a nucleon reads for the process $N\left(P_{1}\right)+\Gamma^{*}\left(q_{1}\right) \rightarrow N\left(P_{2}\right)+\Gamma\left(q_{2}\right)$

$$
\begin{aligned}
& -\left.i \int \frac{d z^{-}}{2 \pi} e^{i x P^{+} z^{-}}\left\langle P_{2}\left|\bar{q}\left(-\frac{1}{2} z\right) \sigma^{+j} \gamma_{5} q\left(\frac{1}{2} z\right)\right| P_{1}\right\rangle\right|_{z^{+}=0, z_{\perp}=0} \\
= & \frac{-i}{P^{+}}\left[H_{T q}(x, \xi, t) \bar{N}\left(P_{2}\right) \sigma^{+j} \gamma_{5} N\left(P_{1}\right)+E_{T q}(x, \xi, t) \bar{N}\left(P_{2}\right) \frac{\varepsilon^{+j \alpha \beta} \Delta_{\alpha} \gamma_{\beta}}{2 M} N\left(P_{1}\right)\right. \\
& \left.+\tilde{H}_{T q}(x, \xi, t) \bar{N}\left(P_{2}\right) \frac{\varepsilon^{+j \alpha \beta} \Delta_{\alpha} P_{\beta}}{M^{2}} N\left(P_{1}\right)+\tilde{E}_{T q}(x, \xi, t) \bar{N}\left(P_{2}\right) \frac{\varepsilon^{+j \alpha \beta} P_{\alpha} \gamma_{\beta}}{2 M} N\left(P_{1}\right)\right]
\end{aligned}
$$

with $\Delta_{\mu}=q_{1 \mu}-q_{2 \mu}, t=\Delta^{2}, P_{\mu}=\left(P_{1 \mu}+P_{2 \mu}\right) / 2, n_{\mu}=(1,0,0,-1) /\left(\sqrt{2} P^{+}\right)$, hence $n \cdot P=1$, and $\xi=-n \cdot \Delta / 2$. An OPE analysis shows that the n'th moment of a GPD is a polynomial in $\xi$ of at most order $n$. The coefficients are functions of the remaining variable $t$ and are called Generalized Form Factors (GFFs), in our case

$$
H_{T}^{n}(\xi, t)=\int_{-1}^{1} d x x^{n-1} H_{T}(x, \xi, t)
$$

$$
\begin{array}{llrl}
H_{T}^{n=1}(\xi, t) & =A_{T 10}(t)=g_{T}(t), & & H_{T}^{n=2}(\xi, t)=A_{T 20}(t), \\
\widetilde{H}_{T}^{n=1}(\xi, t)=\widetilde{A}_{T 10}(t), & & \widetilde{H}_{T}^{n=2}(\xi, t)=\widetilde{A}_{T 20}(t), \\
E_{T}^{n=1}(\xi, t) & =B_{T 10}(t), & & E_{T}^{n=2}(\xi, t)=B_{T 20}(t), \\
\widetilde{E}_{T}^{n=1}(\xi, t) & =\widetilde{B}_{T 10}(t)=0, & & \widetilde{E}_{T}^{n=2}(\xi, t)=(-2 \xi) \widetilde{B}_{T 21}(t) .
\end{array}
$$

These GFFs are related to matrix elements according to

$$
\begin{aligned}
A_{[\mu v]} S_{\left\{v \mu_{1}\right\}}\left\langle P_{2} \Lambda^{\prime}\left|\bar{\psi}(0) i \sigma^{\mu v} \stackrel{\leftrightarrow}{D}^{\mu_{1}} \psi(0)\right| P_{1} \Lambda\right\rangle & =A_{[\mu v]} S_{\left\{v \mu_{1}\right\}} \bar{U}\left(P_{2}, \Lambda^{\prime}\right)\left\{i \sigma^{\mu v} P^{\mu_{1}} A_{T 20}(t)\right. \\
& +\frac{P^{[\mu} \Delta^{v]}}{M^{2}} P^{\mu_{1}} \widetilde{A}_{T 20}(t)+\frac{\gamma^{[\mu} \Delta^{v]}}{2 M} P^{\mu_{1}} B_{T 20}(t) \\
& \left.+\frac{\gamma^{[\mu} P^{v]}}{M} \Delta^{\mu_{1}} \widetilde{B}_{T 21}(t)\right\} U\left(P_{1}, \Lambda\right),
\end{aligned}
$$


To illustrate the meaning of these functions let us quote a result from [3] for the corresponding impact parameter $\left(b_{\perp}\right)$ dependent GPDs in the transverse plane:

$$
\begin{aligned}
& \left.\frac{1}{(2 \pi)^{2}} \int d^{2} \Delta_{\perp} \mathrm{e}^{\mathrm{i} b_{\perp} \cdot \Delta_{\perp}} \int \frac{d z^{-}}{2 \pi} e^{i x \bar{P}^{+} z^{-}}\left\langle P_{2}\left|\bar{q}\left(-\frac{1}{2} z\right) \gamma^{+}\left[1+\vec{s} \cdot \vec{\gamma} \gamma_{5}\right] q\left(\frac{1}{2} z\right)\right| P_{1}\right\rangle\right|_{z^{+}=0, z_{\perp}=0} \\
= & \frac{1}{2}\left[H-S^{i} \varepsilon^{i j} b^{j} \frac{1}{m} E^{\prime}-s^{i} \varepsilon^{i j} b^{j} \frac{1}{m}\left(E_{T}^{\prime}+2 \tilde{H}_{T}^{\prime}\right)\right. \\
& \left.+s^{i} S^{i}\left(H_{T}-\frac{1}{4 m^{2}} \Delta_{b} \tilde{H}_{T}\right)+s^{i}\left(2 b^{i} b^{j}-b^{2} \delta^{i j}\right) S^{j} \frac{1}{m^{2}} \tilde{H}_{T}^{\prime \prime}\right]
\end{aligned}
$$

with $E_{T}^{\prime}=\frac{\partial}{\partial b^{2}} E_{T}, \tilde{H}_{T}^{\prime \prime}=\left(\frac{\partial}{\partial b^{2}}\right)^{2} \tilde{H}_{T}$. As nonzero $b_{\perp}$-dependent terms can only appear due to orbital angular momentum these terms have a straight-forward physical meaning. The second term describes the correlation of nucleon spin and quark orbital angular momentum, the third the correlation of quark spin and quark orbital momentum and the fourth the correlation between nucleon and quark transverse spin.

\section{Simulation and results}

We used the QCDSF/UKQCD configurations with $N_{f}=2$ flavours of dynamical non-perturbatively $\mathscr{O}(a)$ improved Wilson fermions and Wilson glue. Only connected graphs were calculated, which actually should be a very good approximation for helicity flip GPDs. The results were nonperturbatively renormalized (Rome-Southampton method) and are given in the $\overline{M S}$-scheme at a scale of $2 \mathrm{GeV}^{2}$. The resulting GFFs are shown in Fig. 1.

Simple dipole fits to these data work very well and give the fit parameters

$$
\begin{aligned}
& \langle 1\rangle_{\delta}^{u}=A_{T 10}^{u}(0)=0.857 \pm .013, \quad m_{D}=1.732 \pm .036 \mathrm{GeV} \\
& \langle 1\rangle_{\delta}^{d}=A_{T 10}^{d}(0)=-0.212 \pm .005, \quad m_{D}=1.741 \pm .056 \mathrm{GeV} \\
& \langle x\rangle_{\delta}^{u}=A_{T 20}^{u}(0)=0.268 \pm .006, \quad m_{D}=2.312 \pm .071 \mathrm{GeV} \\
& \langle x\rangle_{\delta}^{d}=A_{T 20}^{d}(0)=-0.052 \pm .002, \quad m_{D}=2.448 \pm .173 \mathrm{GeV} .
\end{aligned}
$$

From the first moments we can deduce the much discussed isovector tensor charge

$$
\langle 1\rangle_{\delta}^{u}-\langle 1\rangle_{\delta}^{d}=1.068 \pm 0.016
$$

This results agrees on the 5-15\% level with some previous lattice results in the quenched approximation [5]. The importance of this number lies in the fact that the difference between the tensor charge and $g_{A}$ tests directly the effects due to different orientations of the nucleon spin relative to the boost direction. (In the absence of any such effects it would be zero.) The tensor charge has, therefore, been the subject of numerous model calculations. We find only a very mild dependence on the pion mass, as illustrated in Fig. 2.

\section{The Soffer bound}

Just as normal GPDs contain information on the unpolarized and longitudinally polarized quark distributions, $q\left(x, Q^{2}\right)$ and $\Delta q\left(x, Q^{2}\right)$, the tensor GPDs contain information about the third 

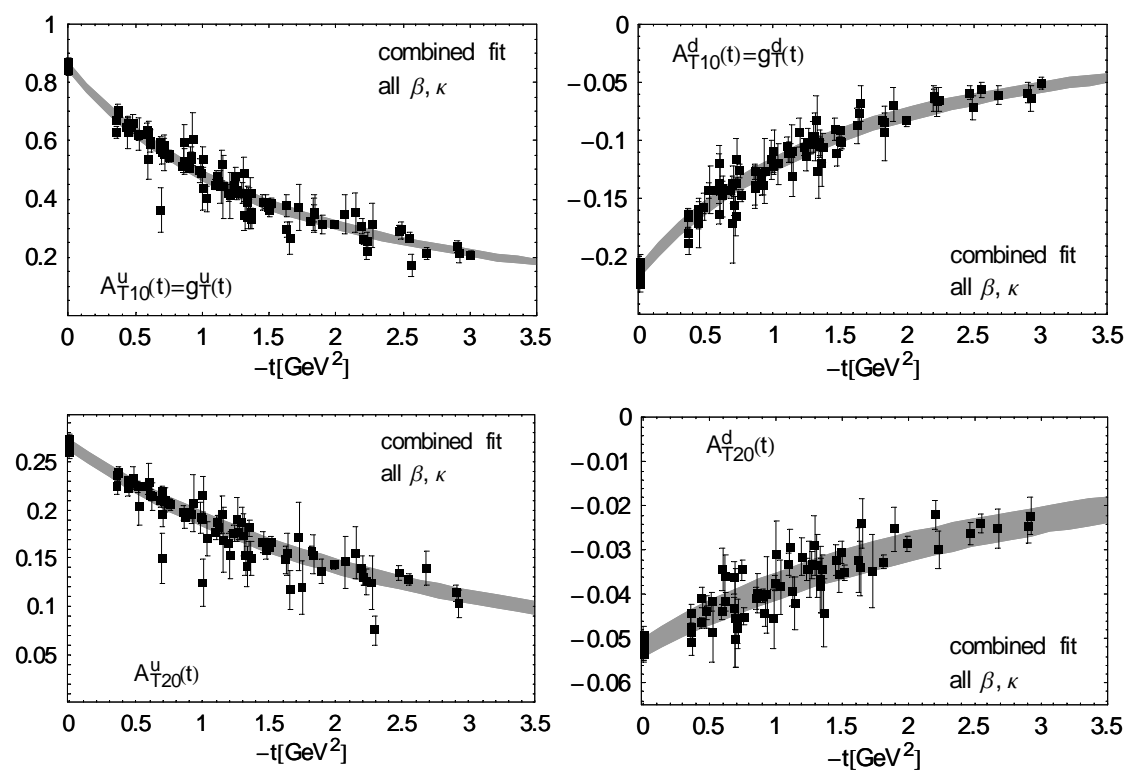

Figure 1: The generalized formfactors $A_{T 10}$ and $A_{T 20}$ together with dipole fits.

twist-2 distribution function, the quark-transversity $\delta q\left(x, Q^{2}\right)$. Transversity is the probability to hit a quark with momentum fraction $x$ and a transverse spin orientation parallel to a transverse nucleon spin minus the probability for the opposite orientation. The determination of this last distribution function is one of the major aims of several large high-energy experiments. Presently the main task is to determine its overall-size. General unitarity arguments lead to the upper bound

$$
|\delta q(x)| \leq \frac{1}{2}(\Delta q(x)+q(x))
$$

This 'Soffer bound' applies to the quark and antiquark distributions separately. The degree to which it is saturated is crucial for the prospects of an experimental determination. Our results do not really allow us to test it, but we did obtain a closely related quantity, which coincides with moments of the quark transversity to the extent that antiquark contributions are negligible.

$$
\frac{2\left|\left\langle x^{n}\right\rangle_{\delta}^{q}-(-1)^{n}\left\langle x^{n}\right\rangle_{\delta}^{\bar{q}}\right|}{\left(\left\langle x^{n}\right\rangle^{q}-(-1)^{n}\left\langle x^{n}\right\rangle^{\bar{q}}+\left\langle x^{n}\right\rangle_{\Delta}^{q}+(-1)^{n}\left\langle x^{n}\right\rangle_{\Delta}^{\bar{q}}\right)}, \quad n=0,1 .
$$

Because there does not exist a gluon transversity $Q^{2}$-evolution does not produce a large transversitysea contribution, in contrast to the unpolarized and longitudinally polarized case. Therefore, the asymmetries to be measured experimentally become very small for small $x$ and consequently it is mainly the large $x$ domain which is important, were neglecting the sea, i.e. disconnected graphs, should be indeed a good approximation. The fact that our ratios are close to 1, see Fig. 3, is thus good news for planned experiments like e.g. PAX at the FAIR $p \bar{p}$ collider. Note also that the ratios are larger for $\langle x\rangle$ than for $\langle 1\rangle$, as expected. 

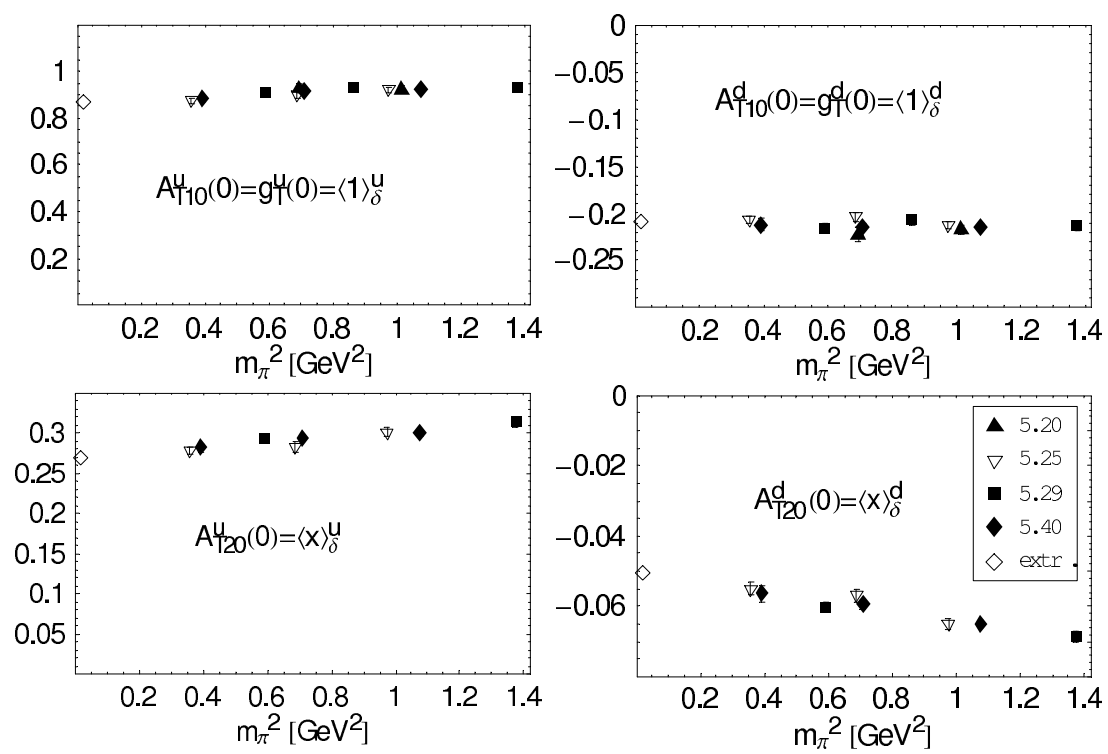

Figure 2: The extrapolated moments at $t=0$ as a function of the pion mass squared.

\section{Conclusions}

Tensor GPDs contain very interesting information on the internal hadron structure, e.g. the quark transversity distributions and the correlations between transverse quark spin, transverse nucleon spin and quark orbital angular momentum, as function of the position in the transverse plane. Our results indicate that these GPDs are not small. QCDSF/UKQCD has performed the first lattice calculations for the lowest $x$-moments of the tensor GPDs. These Generalized Form Factors are well fitted by a dipole form. The resulting value for the tensor charge agrees well with expectations and some earlier quenched lattice results. Results for moments of the tensor GPDs $E_{T}, \tilde{E}_{T}$ and $\tilde{H}_{T}$ are forthcoming.

\section{Acknowledgements}

The numerical calculations have been performed on the Hitachi SR8000 at LRZ (Munich), on the Cray T3E at EPCC (Edinburgh), and on the APEmille at NIC/DESY (Zeuthen). This work is supported in part by the DFG (Forschergruppe Gitter-Hadronen-Phänomenologie) and by the EU Integrated Infrastructure Initiative Hadron Physics under contract number RII3-CT-2004-506078. and by the Helmholtz Association, contract number VH-NG-004.

\section{References}

[1] M. Diehl, Generalized parton distributions, Phys. Rept. 388 (2003) 41, [hep-ph / 0307382 ]. 

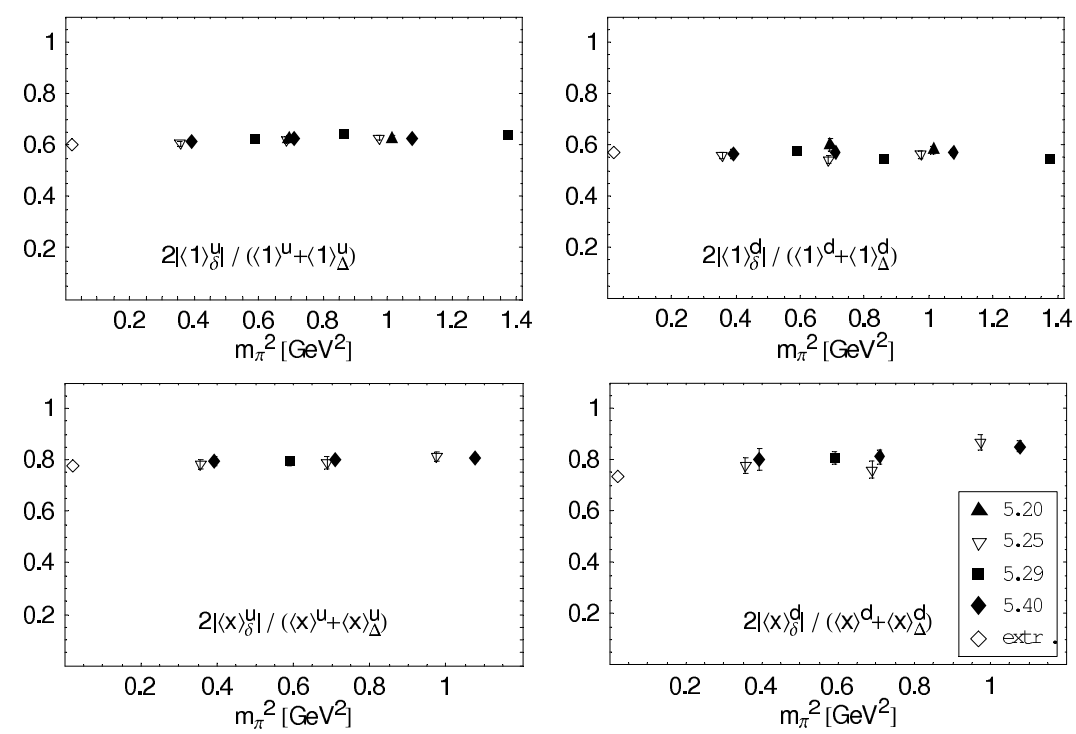

Figure 3: The Soffer-bound-like ratio from Eq. (3.2) for different quark flavours.

[2] M. Göckeler et al. [QCDSF Collaboration], Quark helicity fip generalized parton distributions from two-flavor lattice QCD, [hep-lat/0507001].

[3] M. Diehl and Ph. Hägler, Spin densities in the transverse plane and generalized transversity distributions, [hep-ph/0504175].

[4] J. Soffer, Positivity constraints for spin dependent parton distributions, Phys. Rev. Lett. 74 (1995) $1292[\mathrm{hep}-\mathrm{ph} / 9409254]$.

[5] S. Aoki, M. Doui, T. Hatsuda and Y. Kuramashi, Tensor charge of the nucleon in lattice QCD, Phys. Rev. D 56 (1997) 433 [hep-lat/9608115];

S. Capitani, M. Göckeler, R. Horsley, H. Perlt, D. Petters, D. Pleiter, P.E.L. Rakow, G. Schierholz, A. Schiller and P. Stephenson, Towards a lattice calculation of $\Delta q$ and $\delta q$, Nucl.Phys.Proc.Suppl. 79 (1999) 548-550, [hep-ph/9905573];

K. Orginos, T. Blum and S. Ohta, Nucleon structure functions with domain wall fermions, [hep-lat/0505024];

W. Detmold, W. Melnitchouk and A. W. Thomas, Moments of isovector quark distributions from lattice QCD, Phys. Rev. D 66 (2002) 054501, [hep-lat/ 0206001 ];

D. Dolgov et al. [LHPC collaboration], Moments of nucleon light cone quark distributions calculated in full lattice QCD, Phys. Rev. D 66 (2002) 034506, [hep-lat/ 0201021 ].

[6] C. R. Allton et al. [UKQCD Collaboration], Effects of non-perturbatively improved dynamical fermions in QCD at fixed lattice spacing, Phys. Rev. D65 (2002) 054502, [hep-lat/ 0107021 ]. 